\title{
SCIENTISTS AND ENGINEERS IN CONVERGENCE TECHNOLOGIES IN KOREA: WHERE ARE THEY GOING AND HOW DO THEY COLLABORATE?
}

\author{
Jungmann LEE ${ }^{\mathrm{a}}$, Kiyong OM${ }^{\mathrm{b}}$, Minseok $\mathrm{CHOI}^{\mathrm{c}}$, Chanhoo SONG ${ }^{\mathrm{d}}$, \\ Kwanyoung $\mathrm{KIM}^{\mathrm{d}}$ \\ ${ }^{\mathrm{a}}$ Hoseo University, 165 Sechul-ri, Baebang-myun Asan City, Chungnam Province, Korea \\ ${ }^{\mathrm{b}}$ Korea University of Technology and Education, 307 Gajeon-ri, Byeongcheon-myeon, Dongnam-gu, \\ Cheonan-si, Chungcheongnam-do, Korea \\ 'Electronics and Telecommunications Research Institute (ETRI), Daedeok Science Town, Daejeon, Korea \\ ${ }^{\mathrm{d}}$ Korea Advanced Institute of Science and Technology (KAIST), \\ 373-1 Guseong-dong, Yuseong-gu, Daejeon 305-701, Korea
}

Received 10 August 2012; accepted 12 May 2013

\begin{abstract}
Today convergence technologies have become a major issue in science policy. This paper describes the current state of scientific collaboration in convergence technologies among researchers in South Korea, by conducting survey and the Social Network Analysis (SNA) with a data set of 1,095 researchers who have involved in the development of the convergence technologies. The main research findings are fivefold. First, dominant numbers of researchers are involved in convergence technology with IT because IT is recognized as the most competitive technology in Korea. Second, mobility of researchers is active in convergence technologies. Third, it is found that the researchers in convergence technologies are more productive in terms of the number of research papers per capita than those in other scientific fields. Fourth, they, however, show limited research collaboration, compared with their high productivity. Finally, the members of the network in convergence technologies are closer to each other than those in other scientific fields, but most of their collaborative relationships remain bilateral rather than triangular. Only a few researchers act as hubs, revealing that collaborative research relationship in convergence technologies in Korea is highly concentrated. At the last part, some policy recommendations to promote research collaboration in convergence technologies are discussed.
\end{abstract}

Keywords: convergence technologies, research collaboration, social network analysis, co-authorship analysis.

JEL Classification: O30, O32, O33, O38, O39, I28.

Corresponding author Kwanyoung Kim

E-mail:drucker.kim@kaist.ac.kr,drucker.kim@gmail.com 


\section{Introduction}

The convergence between different technology sectors has emerged as one of the most significant issues in the field of technology development and policy in the $21^{\text {st }}$ century (National Science Technology Committee 2008). Scientists have recently put their efforts on a full convergence of nano-, bio-, and information technologies (hereinafter referred to as NBI convergence technologies), and thanks to their efforts remarkable achievements, albeit in its early stage, have been gained. Especially, IT has had considerable influence on all aspects in the process of technology development. For example, genome project which was originally planned to make up human genetic map is considered as one of major cases, showing convergence between bio technology and IT. In recent years, as researchers in the information security sector had started utilizing human biological characteristics, they have consequently developed an IRIS (Integrated Risk Information System 2013) recognition security system. IRIS is a human health assessment program that evaluates information on health effects that may result from exposure to environmental contaminants.

It is expected that there would be a paradigm shift in understanding in all areas of science, as nano- technology, the study of manipulating matters on an atomic scale, has been remarkably developed (Bainbridge, Roco 2006). In the field of semiconductor they have been able to overcome its physical limits by manufacturing the integrated circuits at a nanometre scale. There has also been a significant change in the medical field since microsurgical robots had emerged. Furthermore, it is anticipated that there would be a remarkable change even in the way people think, as researchers would eventually make materials and machines invisible to the human eye. Likewise, NBI convergence has introduced new technological sectors, such as bio-informatics, and is expected to bring forth inconceivable outcomes in the near future (Roco, Bainbridge 2002).

In the era of convergent technologies, technological competitiveness of a nation will depend on how to secure appropriate human resources and how to organize them (Department of Science and Technology 2007). Most knowledge resides inside the heads of individual researchers. It is also true that there exist some other forms of knowledge, such as the codified knowledge (Cowan et al. 2000; Balconi et al. 2007) saved in computer systems or some resided within individual relationships like organizational culture (Chesbrough 2003). Because most knowledge comes from within the brains of individuals, it is important to improve intellectual skills of researchers. The development of converging technologies in essence requires knowledge of different sectors to be integrated. If a researcher possessed all kinds of knowledge that need to be gained for a convergence technology research, he or she could have conducted the research by him/herself. However, it is a very rare case. Therefore, close collaboration between researchers is needed to share and integrate knowledge resided within the experts working in various sectors (OECD 2004).

This study focuses on collaboration between researchers, one of the core competencies required for researchers in the era of technology convergence. Specifically, it examines the collaborative behaviours of Korean researchers in NIB convergence technologies through the co-authorship analysis. Co-authorship analysis is one of methods for studying collaborative relationships of scientists, and over recent years has been conducted frequently using social 
network analysis (Hereinafter referred to as SNA). In this study collaborative relationships of Korean researchers in the area of converging technologies are identified through co-authorship analysis by using SNA.

This study is structured as follows: Section 1 reviews literatures on scientific collaboration between researchers and SNA. Section 2 explains how data were collected and processed for statistical analysis. Section 3 shows research results about how Korean researchers collaborate in the convergence technology area. Finally, the last section suggests policy recommendations for enhancing collaboration between convergence technology researchers and discusses limitations of this research.

\section{Theoretical background}

\subsection{Scientific collaboration in convergent technologies}

Based on understanding importance of knowledge sharing across disciplines, Gorman (2002) went further, and came up with the concept of trading zone. According to Gorman and Groves (2005), a process to accord is necessary in an organization if there is a difference in individual perceptions. Some collective external events, changes in the subjects that can affect groups such as market, customer, suppliers, and government, may create collective perceptions that will be shared by individuals. Gibbons et al. (1994) categorized the method of knowledge creation into the two knowledge modes, mode 1 and mode 2 knowledge. Mode 1 knowledge designates the knowledge formed in a certain discipline while Mode 2 designates the knowledge formed through inter-disciplinarity or trans-disciplinarity that centred on application area. With the complexity of society, the development of science and technology and the development of transportation, information and telecommunication, Mode 2 knowledge creation is speeding up. Furthermore, Nowotny et al. (2003) asserted that Mode 1 knowledge is an old paradigm of scientific discovery and defined it as a hegemony of theoretical experimental science confined in a separation among disciplines. Meanwhile, they argued that Mode 2 knowledge is a new paradigm for knowledge creation, and it will replace the old paradigm.

To figure out how scientists and engineers share and exchange knowledge in convergence technology, Hwang (2008) reviewed the in-depth interview result with quantum code system researchers in Korea who try to combine code system of IT based medical-engineering field and IT network with quantum physics, and try to combine that to the knowledge. Empirical results showed that the quantum code system could be regarded as a research with depth for new area, while IT based medical-engineering could be a research with width by combining existing knowledge. The research for quantum code system is in the area of deeper in-depthresearch than existing research areas by exchanging and combining the researches of all the areas in which science is at the centre for resolving technological problems. On the contrary, IT based medical-engineering field can be regarded as a research field with width in which loosely related areas in the past are being combined for one purpose.

Generally, researchers work together to achieve a common goal, generating new scientific knowledge. It comes with various cases according to the degree to which they closely collaborate. For example, in one case all collaboration members could participate in the entire 
process of research and contribute directly to the research output. On the contrary, in high energy physics, hundreds of researchers, most of whom do not know one another, take part in the same research project, but each member's participation period and contribution is much limited (Katz, Martin 1997).

Actually, research collaboration includes a diverse range of cases: direct participation throughout the whole process of research, critical contribution to the research output, writing a research proposal together, participation in the verification stage (for example, devising experimental equipment, conducting an experiment, and analysis and explanation of experiment data), research idea generation, providing a theoretical background, supporting research funds, etc.

In this study, we suppose that two researchers are in relations of research collaboration when they co-authored a research paper. Especially in academia, co-authorship is the most visible and available indicator of scientific collaboration and has thus been frequently employed to analyse collaborative behaviours (Abbasi et al. 2010; Milojevic 2010). Therefore, it is reasonable that co-authorship is used as a valid indicator of research collaboration among scientists. Moreover, over the years, not a few researchers have referred to the co-authorship analysis in order to explain how research collaborations work in science. For example, Abbasi et al. (2012) examined the association between scholars' co-authorship network properties and their citation-based research performance using publication data in the field of information science and library science. Dunn et al. (2012) also tried to measure the relative influence of industry authors in comparison to their counterparts in collaborative research networks for clinical trial research using co-authorship.

Recently research collaboration with fellow scientists is on an increasing trend. According to Grossman and Ion (1995), it is found that out of all published papers, the number of papers written by more than two authors has risen as time goes by. For example, in case of Mathematical Review (MR), one of the major journals in mathematics, the portion of papers written by one author has continuously decreased. On the contrary, the portion of papers published by more than two authors has gradually increased. In 1940, the ratio of papers published by one author mounted to over $90 \%$. But in 1993, it has been cut down to less than $60 \%$. On the contrary, the ratio of papers written by more than two authors in the same period had increased to $30 \%$ from $10 \%$.

There are some reasons behind the increase in research collaboration. First, costs of scientific research have sharply soared, especially high costs of buying experimental equipment in the high-tech sector (Katz, Martin 1997). As individual researchers find it difficult to secure all of necessary equipment for doing research, sharing equipment between researchers is mutually beneficial in terms of research productivity. Second, along with costs of purchasing equipment, specialization of knowledge and technology also encourages researchers to collaborate and exchange knowledge. Third, it is also true that costs of exchanging knowledge have been dramatically reduced. A decrease in costs of transportation and telecommunications help researchers to visit one another and to exchange knowledge more easily. Fourth, most researchers hope to work with other researchers to maximize their research performance, because advanced knowledge tends to be created through social interaction between researchers (Katz, Martin 1997). 
By the way, does research collaboration contribute to the improvement of scientific achievement of researchers? According to Lotka (1926), the number of scientists who have published a certain number of papers is inversely proportional to the square of the number of papers. Moreover, the author asserts that the differences of research productivity between scientists are caused by research collaboration. It was empirically found that collaboration with other researchers is directly correlated not only with the number of published papers (Pravdic, Oluic-Vukovic 1986; Abbasi et al. 2011), but also with more sophisticatedly defined measure for the performance of researchers (Abbasi, Altmann 2011; Abbasi et al. 2011). Furthermore, some studies try to measure research performance directly using the results of SNA (Morel et al. 2009).

However, it does not mean that all research collaborations increase productivity regardless of whom the researchers work with. Working with high-productivity researchers leads to an increase in productivity, while collaboration with low-productivity scientists reduces productivity. It was found that articles with more co-authors were cited more frequently and collaborative works were in most cases valued higher by peer researchers (Franceschet, Costantini 2010). Moreover, both citation count and peer rating were enhanced when the affiliations of co-authors were heterogeneous. It was also found that researchers with the best research performance had a greater intensity of international collaboration (Abramo et al. 2011). Therefore, it is important to analyse the pattern of research collaboration of NBI researchers for the improvement of their research productivity.

\subsection{Using social network analysis to study scientific collaboration}

As the network theory is applied to explaining social phenomena, research collaboration is also understood and analysed as a kind of network. According to network theory which is originated from graph theory, a network is defined as a set of vertexes, sometimes called nodes, and edges connecting these vertexes. By the types of vertex, network is can be grouped into physical (e.g. computer network), biological network (e.g. food web, molecular network), and social network (Hereinafter referred to as SN). SN consists of social vertexes such as individuals, organizations, nations and related other entities. The concept of SN has been widely applied in order to examine the structure of relationships between social entities since the concept introduced. SN Analysis using the structural shape and characteristics is initially developed in 1930s (Scott, Carrington 2011), and the term of SN was coined by John Barnes in 1954 (Serrat 2010).

Up to date, the concept of social network has been applied to analysing various social phenomena, and it is called a social network analysis. Researches on social network analysis can be classified into two stages in timeframe. First, in the early stage SN analyses targeted small groups of people. Due to difficulty of securing data, they analysed groups of limited size, such as friends, co-owners or marital relations. As shown in Fig. 1, German researchers influenced by Köhler's 'gestalt theory' and American and British researchers influenced by Radcliffe-Brown started to study the social structure of small size of people. Moreno devised 'sociogram' as a way of representing the social structure with points and lines, and introduced the concept of sociometric 'star', so called key person using sociogram. Warner and Mayo 


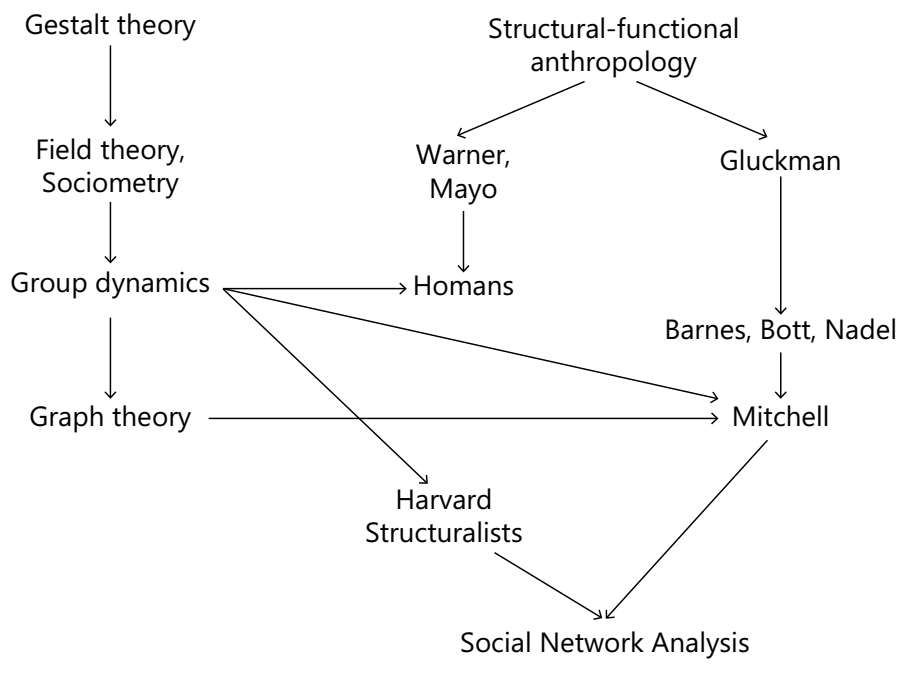

Fig. 1. The lineage of social network analysis Source: Scott 2000.

found 'cliques', which is sub-groups or sub-circles in a social network using sociogram, and Homans identified cliques of a social network by rearranging the pattern of matrix which presents social relationships. Barnes and Bott concerned conflict and power in social relationships, and Nadel focused on the roles of social entities in social network. Mitchell make distinct analysis of ego-centric network from global network (for detail, see Scott 2000). A typical example in this stage is Milgram's experiment, widely known as "six degree" or "small world." He showed in his experiment that any two people in the world can be linked to each other through six steps on an average (Milgram 1967). Even though his experiment was extraordinary at that time, SNA still used data of limited size.

Second, from the early 1970s, At Harvard University, contemporary social network analysis was shaped by the help of the mathematical supports (for detail, see Scott 2000). As the challenge of lack of data has been overcome, researches analysing large-scale social networks have been emerged. A representative case is the studies that analysed co-appearance of movie actors and actresses in the same movies. The research was possible with the advent of the Internet and Internet movie databases. There is also a research analysing social relations of board members of corporations. The subject of this study, research collaboration of scientists also belongs to this category.

For the domain of research and development, Luo and Hsu (2009) identified the star researchers by studying the social network that covered almost all academic researchers of a nation (Taiwan in the study). In addition, individual convergent technologies such as nanotechnology have been often studied using SNA (for example, Zhang et al. 2010). Kim et al. (2007) analysed research collaboration of Korean scientists and engineers using social network analysis. The data set was composed of 2,785 university researchers who took part in research projects supported by the government in 2002. It was supposed that researchers in the same project were on a collaborative relationship. It was found that the network of Korean scientists 
had a structure of "small world" shown in the network of western scientists. Especially, it was very interesting that researchers were closely linked to each other, contrary to the expectation that segmented network would appear in Korea due to special social relations such as regionalism, school relations and kinship. It was also found that a researcher's structural position in the collaboration network had impact on his or her research performance. The authors grouped research projects into six technology areas (information technology (IT), biotechnology (BT), nanotechnology (NT), environmental technology (ET), cultural technology (CT), and space technology (ST)), then analysed research collaboration between these technology areas. While IT researchers were most active in collaboration with other areas, BT researchers played a central role in all kinds of research collaborations.

When there are two or more groups of vertexes in a network, it is called a multi-mode network. For example, a network made up of two groups of men and women is called a twomode network. Meanwhile, a network with only one mode is called a single-mode network. Likewise, edges can be divided into various types. The intensity (strong or weak linkage) and direction (linkage from vertex A to vertex B) of relationship can also be added to edges. When there are differences in the intensity of linkages it is called a valued network, whereas when there are directions in linkages it is called a directed network. Meanwhile, when there is neither intensity nor direction, it is called a simple network. In this study, a SN of scientific collaboration is assumed as a form of simple networks.

\section{Research methods}

\subsection{Data}

The study utilizes two data sets. One is a list of researchers involved in convergence technologies in Korea. The list was obtained from a questionnaire survey of Korean researchers in academia, government-sponsored research institutes, and industry, and includes a total of 1,440 scientists and engineers.

The other data set consists of 307,606 articles in the fields of engineering, natural sciences and medical science selected from the database of Korea Academic Information Corporation, which contains all papers published in Korean journals from 1954 to 2009. This database has information about the title and authors of a paper, the name of journal, and publication year. The number of researchers in the data set is 135,948 , and on average each researcher published 6.09 papers and each paper had 3.06 co-authors.

The intersection of the two data sets was 1,095 researchers, who published at least a paper in the fields of engineering, natural science and medical science in Korean journals during 1954-2009. The rest 345 researchers published no article in three fields in Korean journals during that period because they worked in industry or are experts in other fields.

In previous studies examining research collaboration between scientists using co-authorship information, the first thing they did was to define specific scientific fields to be analysed. For example, Newman (2004) used several database services (MEDLINE, SPIRES, NCSTRL, etc.), which provide information about articles in specific scientific fields, to acquire co-author information. When it is hard to define the scientific field in advance such as nano-technology, 
they first search articles using key words, then conduct network analyses for the resulting articles and co-authors. In case of nano-technology, over $90 \%$ of total articles used in the network analysis were found through a key word "nano" (Li et al. 2007).

However, it was difficult to use the conventional ways mentioned above in this study. First, there is no database around collecting information on articles in convergence technologies. Second, it is hard to collect enough articles by specific key words. When we tried to search articles with key words like "convergence" or "nano," only 100 articles or so were drawn. Due to these obstacles, in the study we first derived experts in convergence technologies and then defined their articles as ones in convergence technologies.

Meanwhile, when analysing a massive amount of article data, the issue of how to correctly identify authors always remains. The reliability of a data set is guaranteed when it is precisely classified by researchers. Most identification errors occur when different researchers have the same name or a researcher uses two or more names. In the former case different researchers are recognized as the same person and this problem takes place frequently in article data with many Asian researchers, while in the latter case a researcher's papers are recognized to be of different researchers by name and this happens a lot when authors' names are written in multiple languages.

However, in the past studies, especially focused on the articles written in English, they did not care about these errors because most people believed that it is rare to find different people with the same name in Western countries and it is also hard to find a person with different names when written in English. It was often supposed that these errors would not affect the result when the portion of Asian researchers is small in the data set (Li et al. 2008).

Nevertheless, this study needs to take into account the problem of different people with the same name because it focuses on articles published in Korean journals and the problem is prevalent in Korean names. The five surnames of Kim, Lee, Park, Choi and Cheong account for more than half of the whole population in Korea. Compared to other Asian countries, Korea has more cases of different people with the same name because she has a unique custom to use common generation characters in names of people.

It is also necessary to consider the problem of the same person with different names in Korean journal articles. Korean journals often publish articles written in English, so it is difficult to match English and Korean names when researchers publish their research in both languages. If we fail to match the English name with the Korean name correctly despite the same person, it is possible to recognize the same person as more than one person. Therefore, it is necessary to find ways to minimize these errors in the first place.

If additional information about authors like their attached organizations, research areas of interest, birthdates, and so on could be used together with name, the problem of different people with the same name would be mitigated substantially. Fortunately, our database provided information about authors' research areas, so name and research area information was used together to identify researchers more correctly. Moreover, all 1,440 researchers identified through our survey in convergence technologies were found by complete enumeration to have different names.

It is also difficult to identify a person correctly when his or her name is written in several languages. Some Korean journals provide an author's name in both Korean and English, and 
some others in either language (Kim et al. 2007; Hwang et al. 2008), thus identification errors may occur. In this study, we could not match Korean names and English names precisely and treated them as different people, so this could be a limitation of the study.

The study processed data by using MS-SQL server in order to extract individual researchers since the massive database contains over 300,000 articles and co-authors are connected in text forms. Then a social network was constructed and analysed with 1,095 vertexes through Pajek, a software for social network analysis. Furthermore, the characteristics of Korean research collaboration in convergence technologies were compared with those of previous studies in other fields.

\subsection{Co-authorship analysis and structural variables of a network}

In a co-authorship network, a vertex indicates a scientist and an edge is constructed when there is a co-authorship relation between two scientists. The simplest form of network research is examining how a scientist collaborates with other researchers, and it is called an ego-centric network (Newman 2001). Grossman and Ion (1995) analysed co-author relationships between Paul Erdös, one of the greatest mathematicians, and his collaborators. They gave 1 to immediate collaborators of the great mathematician, and gave 2 to immediate collaborators of mathematicians with 1, and so on, and then analysed these Erdös numbers.

The number of collaborators assigned the Erdös number of 1 is totalled to 485 and that of researchers who claim the Erdös number of 2 is 5,337. Albert Einstein was assigned the Erdös number of 2, because he had published two papers with Ernst G. Straus, one of his colleagues at the Princeton University, who had published 20 articles collaborating with Paul Erdös. In this way, J. Robert Oppenheimer, one of the leading developers of the US atomic bomb, could claim the Erdös number of 4 . Though he was not an immediate collaborator of Paul Erdös, he was connected to Paul Erdös by passing through 4 steps in the co-authorship network.

Whereas the Erdös number is to analyse a simple tree-shaped network centring around a focal person, there are also other researches examining complicated networks. For example, Hwang et al. (2008) examined how the co-author network was built in an academic journal. After analysing co-author relationships of 242 articles published from 1996 to 2004 in MIS Quarterly, one of the major journals in the field of Management Information Systems, they found that there were three independent sub-networks, each representing a different research subject.

There is also a research examining research collaboration in multiple technology sectors at the same time. Newman (2004) studied research collaborations through the articles published from 1995 to 1999 in the fields of medical science, theoretical physics, high energy physics and computer science. Databases used in the research included MEDLINE (medical science), Los Alamos e-Print Archive (theoretical physics), SPIRES (high energy physics) and NCSTRL (computer science). Each research field was found to have unique collaboration characteristics. In high energy physics, the number of co-authors per paper was much higher than those of other fields. The result reflects the fact that most researches in high energy physics are conducted by collaborative experiments in a large scale. It was also found that $88.7 \%$ of scientists in this field were connected with a network and they were closely linked to each other in consideration of short average distance and high clustering. On the contrary, 
only $57.2 \%$ of all scientists in computer science were linked to the largest network and the rest of them were not connected to it. It was also shown that network members were not close to each other in computer science because both their average path length and maximum path length were higher than those of other fields (Grossman 2002).

From the two previous researches (Newman 2001; Grossman 2002) it is found that the number of paper per researcher is similar for medical science (6.4), theoretical physics (5.1), and mathematics (6.9), but the number of co-authors per paper is quite different from 3.75 (medical science) through 2.53 (theoretical physics) to 1.45 (mathematics). This means that research collaboration in medical science is far more active than in mathematics. Furthermore, the biggest co-authorship network in medical science includes $92 \%$ of a total of $1,520,251$ scientists, while they are $85 \%$ and $82 \%$ in theoretical physics and mathematics respectively. Average path length is the average of distances between all pairs of scientists included in the biggest sub-network. The average path length of medical science was 4.6 even though its network was biggest among the three, compared to 5.9 in theoretical physics and 7.6 in mathematics. Maximum path length, so called network diameter, is the longest of distances between all pairs of scientists in a network. The three fields had similar maximum path lengths from 20 (theoretical physics) to 24 (medical science) and 27 (mathematics).

Clustering coefficient measures the degree to which there is a co-authorship relation between a pair of scientists who are a scientist's collaborators at the same time. This relationship leads to a triangle in the network, and represents the density of connections. Clustering coefficient is calculated as follows:

$\mathrm{C}_{\mathrm{i}}=($ the number of triangles connected to vertex $\mathrm{i}$ ) / (the number of $\mathrm{V}$ shapes with a centre at vertex i).

A V shape with a centre at vertex i means an open triangle with only two sides linking vertex $i$ and other two vertexes respectively (Newman 2003). Interestingly, medical science scored very low at 0.066 , compared to theoretical physics at 0.430 and mathematics at 0.150 . Lastly, with regard to associativity, which measures the degree there is a co-authorship relation between a pair of scientists, both of whom have prolific co-authors, theoretical physics was highest at 0.36 , while medical science and mathematics were 0.13 and 0.12 respectively (Newman 2004).

There is another indicator showing a network characteristic, centrality, which gives a rough indication of the importance of a vertex in the network. In general, there are three methods for measuring centrality. First, the number of co-authors can be used to calculate centrality. This index (degree centrality) takes into account the connectivity of a vertex, allowing a scientist high centrality when he or she has lots of direct collaborators (Newman 2003).

Two other methods are based on the criteria of betweenness and closeness. In order to understand these criteria, it is necessary to understand a concept of shortest path beforehand (Newman 2003). As shown in Figure 1, there are four shortest paths (A-B-C-E-G-J, A-BC-E-H-J, A-B-C-E-I-J, A-B-D-F-I-J) from vertex A to vertex J, passing though 4 vertexes. Betweenness-based centrality $C_{B}(v)$ is calculated by the following equation:

$$
C_{B}(v)=\frac{1}{(n-1)(n-2)} \sum_{s \neq t \neq \sigma \notin v} \frac{\sigma_{s t}(v)}{\sigma_{s t}} .
$$




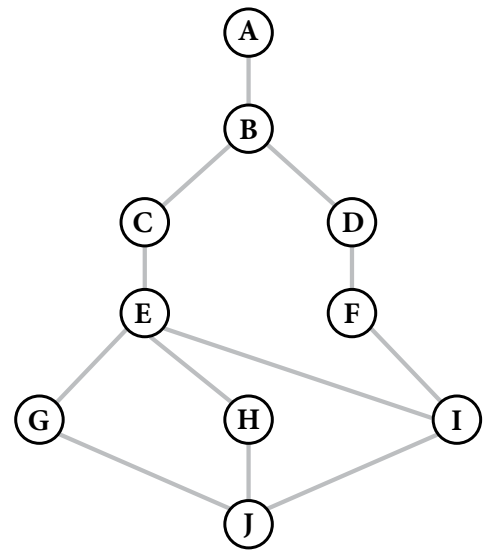

Fig. 2. A network for the explanation of centrality

At first, calculate the number of shortest paths between randomly chosen two vertexes s and $\mathrm{t}\left(\sigma_{\mathrm{st}}\right)$, and the number of shortest paths passing through vertex $v$ among them $\left(\sigma_{s t}(v)\right)$. Then calculate the ratio of $\sigma_{s t}(v)$ to $\sigma_{\text {st }}$, and sum up the ratios of all pairs of vertexes except for vertex $v$. And finally, to offset the effect of network size, the value gained above is normalized by being divided by $(n-1)(n-2)$, where $n$ denotes the number of all vertexes in the network.

For example, in Fig. 2, betweenness-based centrality of vertex $A$ is zero because there is no shortest path passing through vertex $\mathrm{A}$ among the shortest paths between randomly chosen two vertexes from all vertexes except for vertex A. In case of vertex J, betweenness-based centrality is calculated from $(0.5+0.5+0.5)$ / (10-1)(10-2) since vertex $\mathrm{J}$ is located in the middle of G-J-I (0.5), G-J-H (0.5) and H-J-I (0.5). In terms of betweenness-based centrality, vertex J is more significant than vertex $\mathrm{A}$. That is, scientist J plays a more important role than scientist $\mathrm{A}$ in mediating other scientists when they collaborate indirectly through a third scientist. Closeness-based centrality $\mathrm{C}_{\sigma}(v)$ is calculated using the following equation:

$$
C_{\sigma}(v)=\frac{1}{\sum_{t \in \sqrt{v}} d_{G}(v, t)} .
$$

That is, closeness-based centrality of vertex $v$ is a reciprocal of a value gained from summing up all the shortest paths from vertex $v$ to the rest of vertexes in the network $d_{G}(v, t)$ (Newman 2003a, b). For example, in Fig. 2, in case of vertex A the shortest path to vertex B is 1 , to vertexes $C$ and D 2, to vertexes $E$ and $F 3$, to vertexes $G, H$ and I 4, and to vertex J 5, so closeness-based centrality is calculated by $[1 /(1+2+2+3+3+4+4+4+5)]=0.036$. In case of vertex $B$, closeness-based centrality is $[1 /(2+1+2+1+3+2+2+2+3)]=0.056$. It shows that vertex $B$ is easier to reach other vertexes than vertex $A$. Closeness-based centrality indicates how a scientist is closely connected to other scientists in the co-authorship network.

It was shown that all three centrality indicators representing the importance of location in a network have significant influence on both research performance and obtaining research funds. Therefore, this study aims to look into these centrality indicators in the convergence technology research network. This will help understand deeply co-authorship relationships of convergence technology researchers.

The study is different from previous researches in two aspects. First, while previous researchers examined research collaborations in specific journals or within certain scientific sectors, we target research collaborations in NBI convergence technologies, which are characterized as interdisciplinary and heterogeneous.

Second, this study is expected to help understand research collaborations in Korea more effectively and practically. Whereas Kim et al. (2007) analysed research collaboration of 
Korean scientists on the basis of co-participation in government research projects in 2002, we examine co-authorship relationships of scientists using research information databases covering 55 years.

\section{Characteristics of research collaboration in convergence technologies in Korea}

\subsection{The status of scientists and engineers in convergence technologies}

For the survey a list of convergence technology-related research institutions were identified in Korea, then a list of researchers who have been involved in convergence technologies was made by collecting information on them through the organizations they belong to. The participants were researchers participating in convergence technologies in the fields of academia, firms, or research institutes. $43.1 \%$ were working at R\&D institutes, $22 \%$ were working for private companies, and $34.9 \%$ were in academia. While the majority of the survey participants held doctoral degrees (77\%), majority of participants in private firms had master degrees (54.3\%). The data indicates that those who are working on convergence technology tend to have postgraduate education. This finding indicates that more qualified researchers with post-graduate degrees are needed in the convergence technology fields. Among participants, the number of researchers in the IT+BT convergence technologies is biggest (35.4\%) followed by IT+NT (27.8\%), and NT+BT (23.9\%). As IT is recognized as the most competitive technology in Korea, dominant numbers of researchers are involved in convergence technology with IT (63.2\%).

Overall, $49.3 \%$ of research efforts were extended on securing original convergence technologies followed by commercialization of convergence technology (38.3\%) and development of new convergence technology (24.4\%). As expected, R\&D institutes and universities tend to focus more on original technologies, while private companies tend to focus more on commercialization of the existing technologies.

In an attempt to verify the association between two categorical variables for the present study, cross-tabulation analysis was used to determine the way R\&D type differs according to the major (IT, BT or NT) of the participants' undergraduate, graduate or postgraduate course. More specifically, the result showed that researchers with IT majors in their undergraduate schools largely had research experience in convergence technologies that were similar to their present research on the convergence technologies. However, researchers with undergraduate majors in BT or NT as a whole had no experience related to convergence technologies. Researchers with academic backgrounds in IT tend to be more experienced in convergence technology than those of other academic backgrounds. In general, researchers with BT and NT majors didn't have much experience in convergence technology in the past.

According to analysis of major changes (from undergraduate to postgraduate), the majority $(70 \sim 80 \%)$ of the doctoral degree holders had the same majors as their undergraduate degrees. However, some changed their majors, and moved to another academic discipline for their postgraduate degrees. For example, IT majors moved to BT field and NT majors moved to IT field while BT majors did not move to other technology field. 
Let's take a look at sources of convergence technology related knowledge. Overall, researchers acquired their knowledge through collaboration with experts from other organizations (64.1\%) followed by re-training at work (19.6\%) and outsourcing (11.0\%). Researchers, in general, tend to believe intense collaboration $(78.0 \%)$ is more desirable form of collaboration between different disciplines than service collaboration (21.1\%). In here service collaboration is sharing know-how or research equipment. In addition to that, researchers working on the convergence technology tend to believe project-based teams (76.1\%) are more appropriate organizational structure for convergence technology than specialized department $(18.7 \%)$, while they tend to perceive both research teams within organization (51\%) and collaborative teams across organizations (46.5\%) as desirable ways of convergence technology project team composition.

\subsection{Convergence technology researchers' career movement}

More than a half of the convergence technology researchers (67.5\%) had experiences of career movement, and researchers in IT+BT convergence technology tend to experience more career moves in comparison to those in IT+NT and NT+BT convergence technology. This study classified researchers' career movements into seven categories based on their career changes after their original technological backgrounds:

A-1: departmental or organizational changes while maintaining original technological base;

A-2: no changes in departments or organizations;

B-1: adding new technological segment while maintaining original convergence technology they began with;

B-2: exploring various technological segments without firmly maintaining their original convergence technology they began with;

C-1: began with a base technology, currently maintaining a fixed convergence technology;

C-2: began with a base technology, currently exploring various convergence technologies;

D-1: began with a base technology which is not related with the current convergence technology they are working on.

Fig. 3 shows career path classification for convergence technology researchers. Overall, $48.4 \%$ of the convergence technology researchers began their careers with convergence technologies. Among all convergence technology researchers, $18.7 \%$ had departmental or organizational changes (A-1 type), and $29.7 \%$ had no experience of departmental or organizational changes (A-2 type). Meanwhile, researchers who had begun with a base technology but now working on convergence technologies reached $34.9 \%$, while $30.6 \%$ maintain the base technology and work on convergence technologies at the same time (C-1 type), and $4.3 \%$ work on convergence technologies other than the base technology (C-2 type). Eleven percent of convergence technology researchers began with different types of convergence technology. Some $(3.3 \%)$ have added a new convergence technology while maintaining their original convergence technology (B-1 type), and others (7.7\%) have explored a variety of convergence technology fields by modifying their original convergence technology field (B-2 type). Some researchers began with a base technology that was not part of convergence technologies but currently working on convergence technology were $2.4 \%$ of the survey participants (D-1 type). 


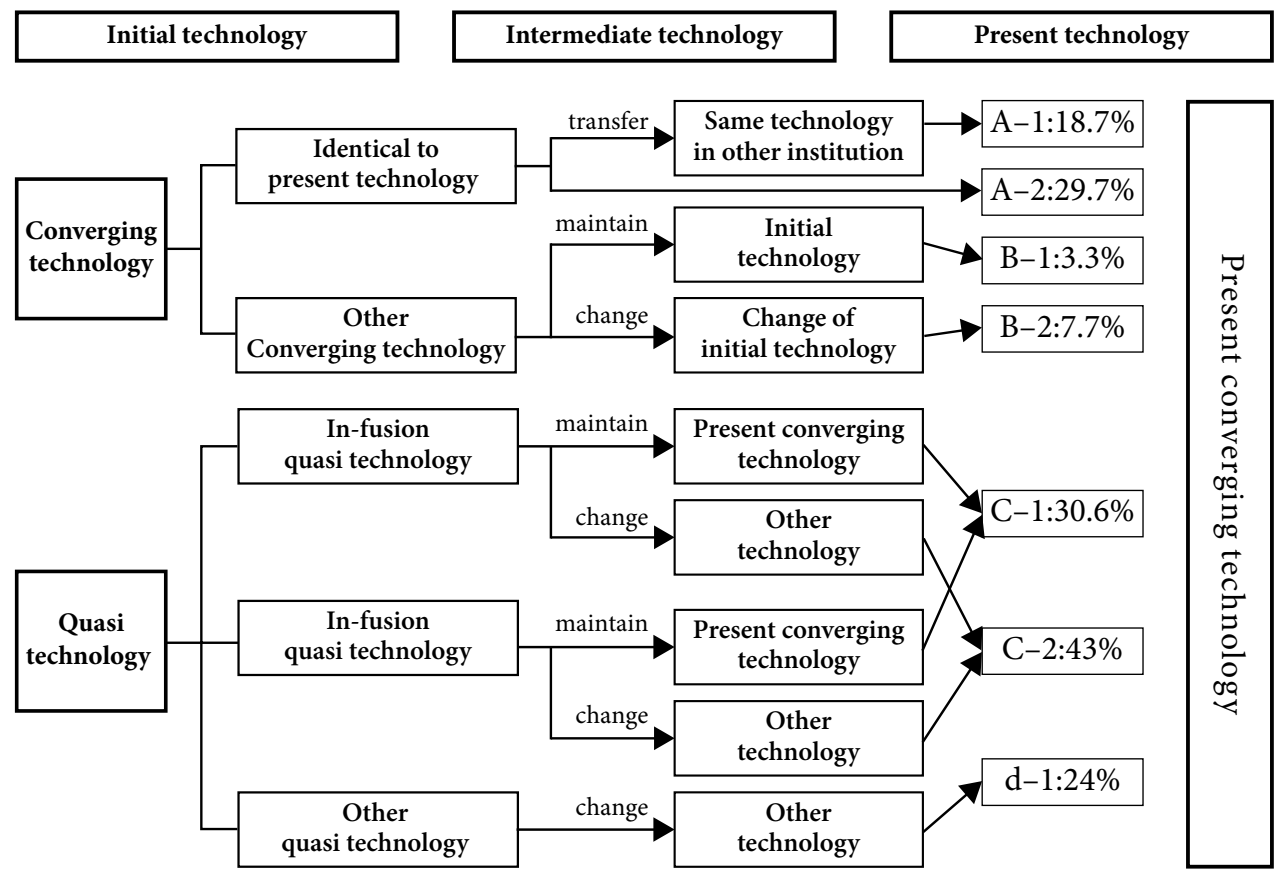

Fig. 3. Career path classification for convergence technology researchers

As can be noted from Table 1, the largest group of researchers in IT+BT convergence technology (36.0\%) began with a base technology (IT or BT), and added new technologies while maintaining the original technologies they began with (C-1 type). 18.7 percent of the researchers had background in IT, while $17.3 \%$ began with BT background. With no change in technological fields, some (26.7\%) had experiences of moving departments and/or organizations (A-1 type), while others (21.3\%) had no experiences of moving (A-2 type).

Table 1. Career movements in the IT+BT convergence technology

\begin{tabular}{|c|c|c|c|c|c|}
\hline Present & Changed type & Percent & Initial tech. & Changes in technological categories & $\%$ \\
\hline \multirow{14}{*}{$\mathrm{IT}+\mathrm{BT}$} & A-1 & 26.7 & $\mathrm{IT}+\mathrm{BT}$ & $\mathrm{IT}+\mathrm{BT} \rightarrow \mathrm{IT}+\mathrm{BT}$ & 26.7 \\
\hline & A-2 & 21.3 & $\mathrm{IT}+\mathrm{BT}$ & $\mathrm{IT}+\mathrm{BT}$ & 21.3 \\
\hline & B-1 & 1.3 & $\mathrm{IT}+\mathrm{NT}$ & $\mathrm{IT}+\mathrm{NT} \rightarrow \mathrm{IT}+\mathrm{BT}+\mathrm{NT} \rightarrow \mathrm{IT}+\mathrm{BT}$ & 1.3 \\
\hline & \multirow[t]{4}{*}{ B-2 } & \multirow[t]{4}{*}{5.3} & $\mathrm{IT}+\mathrm{BT}+\mathrm{MT}$ & $\mathrm{IT}+\mathrm{BT}+\mathrm{MT} \rightarrow \mathrm{BT} \rightarrow \mathrm{IT}+\mathrm{BT}$ & 1.3 \\
\hline & & & $\mathrm{IT}+\mathrm{BT}+\mathrm{NT}$ & $\mathrm{IT}+\mathrm{BT}+\mathrm{NT} \rightarrow \mathrm{IT}+\mathrm{BT}$ & 1.3 \\
\hline & & & $\mathrm{IT}+\mathrm{BT}$ & $\mathrm{IT}+\mathrm{BT} \rightarrow \mathrm{IT}+\mathrm{NT} \rightarrow \mathrm{IT}+\mathrm{BT}$ & 1.3 \\
\hline & & & $\mathrm{IT}+\mathrm{NT}$ & $\mathrm{IT}+\mathrm{NT} \rightarrow \mathrm{IT}+\mathrm{BT}$ & 1.3 \\
\hline & \multirow[t]{2}{*}{$\mathrm{C}-1$} & \multirow[t]{2}{*}{36.0} & $\mathrm{BT}$ & $\mathrm{BT} \rightarrow \mathrm{IT}+\mathrm{BT}$ & 17.3 \\
\hline & & & IT & $\mathrm{IT} \rightarrow \mathrm{IT}+\mathrm{BT}$ & 18.7 \\
\hline & \multirow[t]{2}{*}{$\mathrm{C}-2$} & \multirow[t]{2}{*}{2.7} & IT & $\mathrm{IT} \rightarrow \mathrm{BT} \rightarrow \mathrm{IT}+\mathrm{BT}$ & 1.3 \\
\hline & & & IT & $\mathrm{IT} \rightarrow \mathrm{IT}+\mathrm{NT} \rightarrow \mathrm{IT}+\mathrm{BT}$ & 1.3 \\
\hline & \multirow[t]{2}{*}{ D-1 } & \multirow[t]{2}{*}{2.7} & AI & $\mathrm{AI} \rightarrow \mathrm{IT}+\mathrm{BT}$ & 1.3 \\
\hline & & & NT & $\mathrm{NT} \rightarrow \mathrm{IT}+\mathrm{NT} \rightarrow \mathrm{IT}+\mathrm{BT}$ & 1.3 \\
\hline & N/A & 2.7 & $\mathrm{~N} / \mathrm{R}$ & $\mathrm{N} / \mathrm{R}$ & 2.7 \\
\hline
\end{tabular}


While, almost $30 \%$ of those in the IT+NT convergence technology (29.3\%) have added new technologies while maintaining their base technologies that they began with (C-1 type). Among C-1 type career moves, more researchers had basis on IT (19.0\%) than NT (10.3\%). On the other hand, researchers in the BT+NT convergence technology with no experiences of departments and/or organizations changes (A-2 type) were close to a half (48.0\%), and showed relatively lower level in comparison to other convergence technology fields.

In sum, the career movement was more active in the IT+BT convergence technology, and there were more cases of moves from a base technology to a convergence technology (IT or $\mathrm{BT} \rightarrow \mathrm{IT}+\mathrm{BT})$ rather than shifts from one convergence technology to another convergence technology $(\mathrm{IT}+\mathrm{NT} \rightarrow \mathrm{IT}+\mathrm{NT}+\mathrm{BT})$.

\subsection{A social network analysis on research collaboration}

In Fig. 4, it is found that most of convergence technology researchers are concentrated at the centre of the network, and researchers located at the centre are playing an important role in research collaboration. Meanwhile, researchers at the periphery are found to be connected to the largest sub-network located at the centre, but hardly to be linked to each other. Therefore, co-authorship network of researchers in convergence technologies in Korea can be called a concentrated network where most of the linkages are between centre and periphery and a small part of researchers are not linked to the largest sub-network.

From Table 2, the analysis result of this study showed that each researcher in convergence technologies published 43.7 articles on average, showing pretty high research productivity, but had only 5.1 collaborators on average, lower than high energy physics (173.0), medical science (18.1) and theoretical physics (9.7) and higher than mathematics (3.9) and computer science (3.5). In terms of average path length (3.9) and maximum path length (9), members of the NBI network are more closely located to each other than other networks, but connection density between network members is low due to very low clustering coefficient 0.10 .

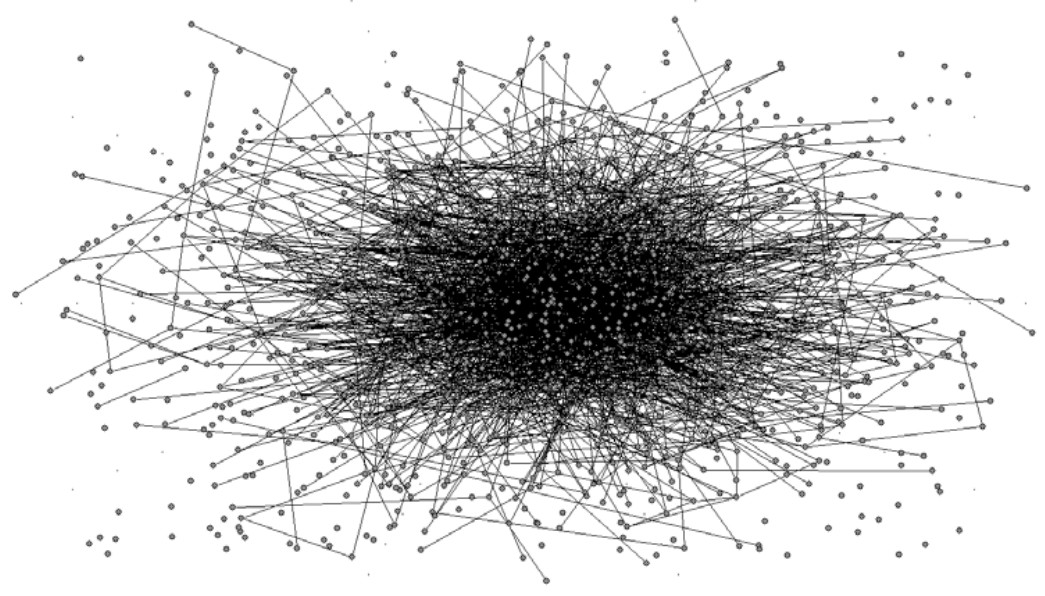

Fig. 4. Co-authorship network in convergence technologies 
Table 2. Comparison of our results with previous studies

\begin{tabular}{|c|c|c|c|c|c|c|}
\hline Category & $\begin{array}{c}\mathrm{NBI} \\
\text { convergence }\end{array}$ & $\begin{array}{l}\text { Medical } \\
\text { science }\end{array}$ & $\begin{array}{c}\text { Theoretical } \\
\text { physics }\end{array}$ & $\begin{array}{l}\text { High energy } \\
\text { physics }\end{array}$ & $\begin{array}{l}\text { Computer } \\
\text { science }\end{array}$ & Mathematics \\
\hline Number of all authors & 1,095 & $1,520,251$ & 52,909 & 56,627 & 11,994 & 253,339 \\
\hline Number of all articles & 47,895 & $2,163,923$ & 98,502 & 66,652 & 13,169 & - \\
\hline $\begin{array}{l}\text { Average number of } \\
\text { articles per author }\end{array}$ & 43.7 & 6.4 & 5.1 & 11.6 & 2.5 & 6.9 \\
\hline $\begin{array}{l}\text { Average number of } \\
\text { authors per article }\end{array}$ & - & 3.7 & 2.5 & 8.9 & 2.2 & 1.4 \\
\hline $\begin{array}{l}\text { Average number of } \\
\text { collaborators per author }\end{array}$ & 5.1 & 18.1 & 9.7 & 173.0 & 3.5 & 3.9 \\
\hline Largest sub-network & - & $92.0 \%$ & $85.0 \%$ & $88.7 \%$ & $57.2 \%$ & $82.0 \%$ \\
\hline Average path length & 3.9 & 4.6 & 5.9 & 4.0 & 9.7 & 7.6 \\
\hline Maximum path length & 9 & 24 & 20 & 19 & 31 & 27 \\
\hline Clustering coefficient & 0.10 & 0.06 & 0.43 & 0.72 & 0.49 & 0.15 \\
\hline Associativity & - & 0.13 & 0.36 & - & - & 0.12 \\
\hline
\end{tabular}

As shown in Table 3, three researchers were publishing papers by themselves with no co-author. The researcher who had the largest number of co-authors collaborated with 42 other researchers, and the next was a researcher working with 33 collaborators. When it comes to degree centrality, showing centrality of vertexes according to the number of co-authors, almost half of all researchers were found to have less than three collaborators.

Table 3. Distribution of the number of co-authors per researcher in the NBI network

\begin{tabular}{ccccc}
\hline $\begin{array}{c}\text { Number of } \\
\text { co-authors }\end{array}$ & Frequency & Ratio & $\begin{array}{c}\text { Cumulative } \\
\text { frequency }\end{array}$ & $\begin{array}{c}\text { Cumulative } \\
\text { ratio }\end{array}$ \\
\hline 0 & 3 & 0.27 & 3 & 0.27 \\
\hline 1 & 281 & 25.67 & 284 & 25.94 \\
\hline 2 & 196 & 17.90 & 480 & 43.84 \\
\hline 3 & 120 & 10.96 & 600 & 54.79 \\
\hline 4 & 101 & 9.22 & 701 & 64.02 \\
\hline 5 & 68 & 6.21 & 769 & 70.06 \\
\hline 6 & 42 & 3.84 & 811 & 74.06 \\
\hline 7 & 35 & 3.20 & 846 & 77.26 \\
\hline 8 & 39 & 3.56 & 885 & 80.82 \\
\hline 9 & 34 & 3.11 & 919 & 83.93 \\
\hline 10 & 28 & 2.56 & 947 & 86.48 \\
\hline 11 & 20 & 1.83 & 967 & 88.31 \\
\hline 12 & 13 & 1.19 & 980 & 89.50 \\
\hline 13 & 16 & 1.46 & 996 & 90.96 \\
\hline 14 & 15 & 1.37 & 1011 & 92.33 \\
\hline 15 & 10 & 0.91 & 1021 & 93.24 \\
\hline 16 & 15 & 1.37 & 1036 & 94.61 \\
\hline
\end{tabular}


Continued Table 3

\begin{tabular}{|c|c|c|c|c|}
\hline $\begin{array}{l}\text { Number of } \\
\text { co-authors }\end{array}$ & Frequency & Ratio & $\begin{array}{l}\text { Cumulative } \\
\text { frequency }\end{array}$ & $\begin{array}{l}\text { Cumulative } \\
\text { ratio }\end{array}$ \\
\hline 17 & 14 & 1.28 & 1050 & 95.89 \\
\hline 18 & 5 & 0.46 & 1055 & 96.35 \\
\hline 19 & 4 & 0.37 & 1059 & 96.71 \\
\hline 20 & 4 & 0.37 & 1063 & 97.08 \\
\hline 21 & 7 & 0.64 & 1070 & 97.72 \\
\hline 22 & 3 & 0.27 & 1073 & 97.99 \\
\hline 23 & 4 & 0.37 & 1077 & 98.36 \\
\hline 24 & 5 & 0.46 & 1082 & 98.81 \\
\hline 25 & 2 & 0.18 & 1084 & 99.00 \\
\hline 26 & 3 & 0.27 & 1087 & 99.27 \\
\hline 27 & 3 & 0.27 & 1090 & 99.54 \\
\hline 28 & 0 & 0.00 & 1090 & 99.54 \\
\hline 29 & 1 & 0.09 & 1091 & 99.63 \\
\hline 30 & 2 & 0.18 & 1093 & 99.82 \\
\hline 31 & 0 & 0.00 & 1093 & 99.82 \\
\hline 32 & 0 & 0.00 & 1093 & 99.82 \\
\hline 33 & 1 & 0.09 & 1094 & 99.91 \\
\hline 34 & 0 & 0.00 & 1094 & 99.91 \\
\hline 35 & 0 & 0.00 & 1094 & 99.91 \\
\hline 36 & 0 & 0.00 & 1094 & 99.91 \\
\hline 37 & 0 & 0.00 & 1094 & 99.91 \\
\hline 38 & 0 & 0.00 & 1094 & 99.91 \\
\hline 39 & 0 & 0.00 & 1094 & 99.91 \\
\hline 40 & 0 & 0.00 & 1094 & 99.91 \\
\hline 41 & 0 & 0.00 & 1094 & 99.91 \\
\hline 42 & 1 & 0.09 & 1095 & 100.00 \\
\hline
\end{tabular}

Along with degree centrality, there are also betweenness-based centrality and closeness-based centrality, showing the importance of a vertex's location in a network. In the study, two indices on average were 0.001 and 0.139 respectively. In terms of betweenness-based centrality in Table 4, two researchers are playing a very important role in bridging other researchers. If the two researchers did not exist, it could be possible that the average path length increases rapidly or lots of linkages are broken off. On the contrary, it is found in Table 5 that a lot of vertexes are having similar values of closeness-based centrality.

Table 4. Distribution of betweenness-based centrality in the NBI network

\begin{tabular}{ccccc}
\hline $\begin{array}{c}\text { Betweenness-based } \\
\text { centrality }\end{array}$ & Frequency & Ratio & $\begin{array}{c}\text { Cumulative } \\
\text { frequency }\end{array}$ & $\begin{array}{c}\text { Cumulative } \\
\text { ratio }\end{array}$ \\
\hline 0.0000 & 538 & 49.13 & 538 & 49.13 \\
\hline $0.0000-0.0134$ & 540 & 49.32 & 1078 & 98.45 \\
\hline $0.0134-0.0268$ & 15 & 1.37 & 1093 & 99.82 \\
\hline $0.0268-0.0402$ & 2 & 0.18 & 1095 & 100.00 \\
\hline
\end{tabular}


Table 5. Distribution of closeness-based centrality in the NBI network

\begin{tabular}{ccccc}
\hline $\begin{array}{c}\text { Closeness-based } \\
\text { centrality }\end{array}$ & Frequency & Ratio & $\begin{array}{c}\text { Cumulative } \\
\text { frequency }\end{array}$ & $\begin{array}{c}\text { Cumulative } \\
\text { ratio }\end{array}$ \\
\hline 0.0000 & 283 & 25.84 & 283 & 25.84 \\
\hline $0.0000 \sim 0.0882$ & 16 & 1.46 & 299 & 27.31 \\
\hline $0.0882 \sim 0.1764$ & 228 & 20.82 & 527 & 48.13 \\
\hline $0.1764 \sim 0.2646$ & 568 & 51.87 & 1095 & 100.00 \\
\hline
\end{tabular}

Table 6 shows important vertexes from a centrality point of view. A total of nine researchers are ranked within upper 10 in all three centrality criteria simultaneously, and they play important roles in the network of 1,095 researchers. Especially, two researchers (identification numbers 9 and 13) are crucially significant, acting as core leaders in developing convergence technologies in Korea.

Table 6. Important vertexes from the centrality perspective in the NBI network

\begin{tabular}{cccc}
\hline $\begin{array}{c}\text { Identification } \\
\text { number of vertex }\end{array}$ & $\begin{array}{c}\text { Number of } \\
\text { collaborators }\end{array}$ & $\begin{array}{c}\text { Betweenness-based } \\
\text { centrality }\end{array}$ & $\begin{array}{c}\text { Closeness-based } \\
\text { centrality }\end{array}$ \\
\hline 9 & 42 & 0.040 & 0.265 \\
\hline 13 & 33 & 0.029 & 0.263 \\
\hline 27 & 30 & 0.018 & 0.251 \\
\hline 32 & 30 & 0.021 & 0.254 \\
\hline 18 & 29 & 0.015 & - \\
\hline 30 & 27 & 0.017 & 0.250 \\
\hline 59 & 27 & 0.016 & 0.247 \\
\hline 17 & 27 & 0.016 & 0.248 \\
\hline 12 & 26 & - & 0.250 \\
\hline 38 & 26 & - & 0.246 \\
\hline 33 & - & 0.016 & - \\
\hline 36 & - & 0.015 & - \\
\hline 34 & - & - & 0.249 \\
\hline
\end{tabular}

\section{Discussions and conclusions}

From the survey and social network analysis of Korean researchers in convergence technologies, the following five characteristics were found. First, dominant numbers of researchers are involved in convergence technology with IT because IT is recognized as the most competitive technology in Korea. And researchers with academic backgrounds in IT tend to be more experienced in convergence technology than researchers with BT and NT majors.

Second, mobility of researchers is active in the field of convergence technologies in Korea. More than a half of the convergence technology researchers $(67.5 \%)$ had experiences of career movement, and researchers in IT+BT convergence technology $(75.7 \%)$ tend to experience more career moves in comparison to those in IT+NT and NT+BT convergence technology. 
Third, the number of articles per person of convergence technology researchers is much higher than that of researchers in other scientific fields, although only Korean journals were considered in the study. It shows that they have high research productivity as far as articles in Korean journals are concerned and actively create new knowledge in convergence technologies.

Fourth, the number of collaborators per person was relatively low, compared to the high research productivity. It means that most Korean convergence technology researchers do not actively involve in research collaboration with other researchers. There could be some reasons for the limited co-authorships. One of them would be the fact that sometimes researchers find difficulty in co-authoring papers with researchers in different fields because they have their own specialized and different methods of experiment, analysis and writing. More in-depth researches are needed in this regard in the future.

Finally, the distance between convergence technology researchers is found to be closer than that in other scientific fields, but their connection density is quite low. That is, collaborations between researchers usually take place in a bilateral way, rather than in a triangular way. In addition, it is also found that a small number of researchers have crucial influence on the whole network in Korea.

There seems to be some cultural influences behind the lack of triangular collaborations. The prevalence of relationships in Korea based on regionalism, school ties, blood relations, and age groups is obviously expected to restrict the formation of a diverse range of collaborative relationships and ultimately to curb the opportunity of advancing in the global competition. Therefore, it is recommended for the government to make an effort to provide policy measures to promote research collaboration in convergence technologies.

First of all, it is necessary to consider enhancing mobility of researchers from one organization to another. The transfer of knowledge is made not only through education and training but through movement of researchers themselves. However, it is not easy for Korean researchers to move to another organization. For example, if a professor moves to another university in the middle of conducting a research project, various regulations prohibit him or her from continuing the project in new university. Moreover, in this case dedicated researchers to the project or graduate students who work with the professor cannot move to the university together.

Expansion and reproduction of knowledge tend to be made through collaboration of researchers from various groups. Especially, in the era of convergence technologies horizontal knowledge integration is much more important than vertical knowledge transmission, and quality of communications between researchers in different scientific fields has great impact on research performance. Recently, with the rapid increase of $\mathrm{R} \& \mathrm{D}$ globalization, cooperation with foreign researchers has become a critical issue in science and technology policies. It is also necessary to effectively translate technical terminology of convergence technologies in foreign languages into Korean, not to mention the importance of English. To make it possible, it is suggested to form a collaboration network between linguists, information system experts and scientists and engineers from all kinds of technical fields.

Even though it is a very important topic to investigate research collaboration in convergence technologies, there has been short of related researches. In this respect, this study is expected to shed some light on researchers and policy makers involved in convergence technologies. Nevertheless, the study has several limitations. First, the number of vertexes 
in our social network is not large enough compared to other social network analyses. Thus, it is necessary to acquire a larger list of convergence technology researchers to get more meaningful results from empirical analysis. Second, this study assumes the network of scientific as a simple network consisting only binary relationships between two researchers. In other words, this study does not differentiate how many times two researchers co-authored. This assumption can regard hundred times and just one time of collaboration as the same thing. Therefore, this study can be extended by adopting a valued network analysis in order to test with a more realistic model. Third, the risk of identification errors was not completely eliminated in the study. There are possibilities of reflecting a reality incorrectly by regarding different researchers with the same name as the same person in spite of having given consideration to research interest of researchers additionally and failing to recognize as the same person when a researcher uses Korean name and English name separately. More attention and efforts are needed in the future in order to address this problem. Fourth, the study assumes that all articles of a researcher in convergence technologies are related to convergence technologies. But, a more probable scenario is that early research interest of a researcher was in one of the conventional scientific fields, and as time goes by it was expanded or converted to a new field like convergence technologies. Therefore, analysis results of the study should be interpreted with caution because the articles used in the analysis were not sifted in consideration of their themes.

\section{Acknowledgement}

This research was partially supported by the Korea Research Foundation(KRF) under the Basic Research Support Program (Basic Science, Collaboration Research) and also partially supported by the MEST (The Ministry of Education, Science and Technology), Korea. Project number is D00001.

\section{References}

Abbasi, A.; Altmann, J. 2011. On the correlation between research performance and social network analysis measures applied to research collaboration networks, in The 44th Annual Hawaii International Conference on System Sciences (HICSS), 4-7 January, 2011, Waikoloa, HI, USA, 4-7.

Abbasi, A.; Altmann, J.; Hossain, L. 2011. Identifying the effects of co-authorship networks on the performance of scholars: a correlation and regression analysis of performance measures and social network analysis measures, Journal of Informetrics 5(4): 594-607. http://dx.doi.org/10.1016/j.joi.2011.05.007

Abbasi, A.; Altmann, J.; Hwang, J. 2010. Evaluating scholars based on their academic collaboration activities: two indices, the RC-index and the CC-index, for quantifying collaboration activities of researchers and scientific communities, Scientometrics 83(1): 1-13. http://dx.doi.org/10.1007/s11192-009-0139-2

Abbasi, A.; Chung, K.; Hossain, L. 2012. Egocentric analysis of co-authorship network structure, position and performance, Information Processing and Management 48(4): 671-679. http://dx.doi.org/10.1016/j.ipm.2011.09.001

Abramo, G.; D’Angelo, C. A.; Solazzi, M. 2011. Are researchers that collaborate more at the international level top performers? An investigation on the Italian university system, Journal of Informetrics 5(1): 204-213. http://dx.doi.org/10.1016/j.joi.2010.11.002 
Bainbridge, W. S.; Roco, M. C. 2006. Managing nano-bio-info-cogno innovations: converging technologies in society. Berlin: Springer. 396 p. http://dx.doi.org/10.1007/1-4020-4107-1

Balconi, M.; Pozzali, A.; Viale, R. 2007. The 'Codification Debate' revisited: a conceptual framework to analyze the role of tacit knowledge in economics, Industrial and Corporate Change 16(5): 823-849. http://dx.doi.org/10.1093/icc/dtm025

Chesbrough, H. W. 2003. Open innovation. Boston: Harvard Business School Press. 375 p.

Cowan, R.; David, P. A.; Foray, D. 2000. The explicit economics of knowledge codification and tacitnesss, Industrial and Corporate Change 9(2): 211-253. http://dx.doi.org/10.1093/icc/9.2.211

Department of Science and Technology (DST). 2007. Convergence Technology Development Basic Plan in Korea. Dept. of Science and Technology, Korea (in Korean).

Dunn, A.; Gallego, B.; Coiera, E. 2012. Industry influenced evidence production in collaborative research communities: a network analysis, Journal of Clinical Epidemiology 65(5): 535-543. http://dx.doi.org/10.1016/j.jclinepi.2011.10.010

Franceschet, M.; Costantini, A. 2010. The effect of scholar collaboration on impact and quality of academic papers, Journal of Informetrics 4(4): 540-553. http://dx.doi.org/10.1016/j.joi.2010.06.003

Gibbons, M.; Limoges, C.; Nowotny, H.; Schwartzman, S.; Scott, P.; Trow, M. 1994. The new production of knowledge: the dynamics of science and research in contemporary societies. London: Sage. $179 \mathrm{p}$.

Gorman, M. E. 2002. Comment-levels of expertise and trading zones, Social Studies of Science 32(5/6): 933-938. http://dx.doi.org/10.1177/030631202128967343

Gorman, M. E.; Groves, J. 2005. Collaboration on convergence technologies: education and practice, in Bainbridge, W. S.; Roco, M. C. (Eds.). Managing Nano-Bio-Info-Cogno innovations: converging technologies in society. National Science Foundation, 71-81.

Grossman, J. W. 2002. The evolution of the mathematical research collaboration graph, Congress Numerantium 158: 202-212.

Grossman, J. W.; Ion, P. D. F. 1995. On a portion of the well-known collaboration graph, Congress Numerantium 108: 129-131.

Hwang, G. 2008. A program to cultivate specialized manpower for promoting convergence based on IT, Information and Communications Magazine 25(11): 42-47 (in Korean).

Hwang, M.; Ahn, J.; Chang, J. 2008. A study on the pattern of collaboration networks: focused on the co-authorship analysis of MIS Quarterly, Journal of Korean Electronic Commerce Society 13(4): 193-207 (in Korean).

Integrated Risk Information System (IRIS) [online], [cited 6 March 2013]. Available from Internet: http:// www.epa.gov/IRIS/

Katz, J. S.; Martin, B. R. 1997. What is research collaboration?, Research Policy 26(1): 1-18. http://dx.doi.org/10.1016/S0048-7333(96)00917-1

Kim, Y.; Yoon, J.; Cho, H.; Kim, Y. 2007. Network structure of collaborative researches in science and technology: small world and position effect, Korean Sociology Journal 41(4): 68-103 (in Korean).

Li, X.; Chen, H.; Dang, Y.; Lin, Y.; Larson, C. A.; Roco, M. C. 2008. A longitudinal analysis of nanotechnology literature: 1976-2004, Journal of Nanoparticle Research 10(1): 3-22. http://dx.doi.org/10.1007/s11051-008-9473-1

Li, X.; Lin, Y.; Chen, H.; Roco, M. C. 2007. Worldwide nanotechnology development: a comparative study of USPTO, EPO, and JPO patents (1976-2004), Journal of Nanoparticle Research 9(6): 977-1002. http://dx.doi.org/10.1007/s11051-007-9273-z

Lotka, A. J. 1926. The frequency distribution of scientific productivity, Journal of Washington Academy of Science 16: 317-323. 
Luo, Y.; Hsu, C. 2009. An empirical study of research collaboration using social network analysis, in IEEE International Conference on Computational Science and Engineering, 2009 (ICCSE '09), 29-31 August, 2009, Vancouver, Canada 4: 921-926. http://dx.doi.org/10.1109/CSE.2009.253

Milgram, S. 1967. The small-world problem, Psychology Today 1: 62-67.

Milojevic, S. 2010, Modes of collaboration in modern science: beyond power laws and preferential attachment, Journal of the American Society for Information Science and Technology 61(7): 1410-1423. http://dx.doi.org/10.1002/asi.21331

Morel, C. M.; Serruya, S. J.; Penna, G. O.; Guimarães, R. 2009. Co-authorship network analysis: a powerful tool for strategic planning of research, development and capacity building programs on neglected diseases, PLoS Negleted Tropical Disseases 3(8): e501. http://dx.doi.org/10.1371/journal.pntd.0000501

National Science Technology Committee (NSTC) 2008. Comprehensive Development Program for National Convergence Technology (2009-2013), NSTC, Korea (in Korean).

Newman, M. 2001. The structure of scientific collaboration network, Proceedings of the National Academy of Sciences 98(2): 404-409. http://dx.doi.org/10.1073/pnas.98.2.404

Newman, M. 2004. Coauthorship networks and patterns of scientific collaboration, Proceedings of the National Academy of Sciences 101(Suppl. 1): 5200-5205. http://dx.doi.org/10.1073/pnas.0307545100

Newman, M. E. J. 2003a. Ego-centered networks and the ripple effect, Social Networks 25(1): 83-95. http://dx.doi.org/10.1016/S0378-8733(02)00039-4

Newman, M. E. J. 2003b. The structure and function of complex networks, SIAM Review 45(2): 167-256. http://dx.doi.org/10.1137/S003614450342480

Nowotny, H.; Scott, P.; Gibbons, M. 2003. 'Mode 2' revisited: the new production of knowledge-introduction, Minerva 41(3): 179-94. http://dx.doi.org/10.1023/A:1025505528250

OECD. 2004. Innovation in the knowledge economy, Discussion Paper. Center for Education Research and Innovation.

Pravdic, N.; Oluic-Vukovic, V. 1986. Dual approach to multiple authorship in the study of collaboration/ scientific output relationship, Scientometrics 10(5-6): 259-280. http://dx.doi.org/10.1007/BF02016774

Roco, M. C.; Bainbridge, W. S. 2002. Converging technologies for improving human performance-nanotechnology, biotechnology, information technology and cognitive science. National Science Foundation. $482 \mathrm{p}$.

Scott, J.; Carrington, P. J. 2011. The SAGE handbook of social network analysis. Sage Publications Limited. $640 \mathrm{p}$.

Scott, J. 2000. Social network analysis: a handbook. Sage Publications Limited. 208 p.

Serrat, O. 2010. Social network analysis. Washington, DC: Asian Development Bank.

Zhang, H.; Qiu B.; Lvanova, K.; Giles, C. L.; Foley, H. C.; Yen, J. 2010. Locality and attachedness-based temporal social network growth dynamics analysis: a case study of evolving nanotechnology scientific collaboration networks, Journal of the American Society for Information Science and Technology 61(5): 964-977. http://dx.doi.org/10.1002/asi.21225

Jungmann LEE obtained his PhD in Economics from the City University of New York. He has also served as an Advisor for various projects (IT technology policy and HRD Policy) of the Ministry of Information and Communication, Korea. He is an Associate Professor at the Department of Digital Business at Hoseo University. His research has focused on the areas of technology policy, R\&D management, and the economics of technology innovation at the Electronics and Telecommunications Research Institute.

Kiyong OM received his MS and PhD in Management Technology from the Korea Advanced Institute of Science and Technology (KAIST) in 1993 and 1998, respectively. For over five years, he has been actively involved with the formation and implementation of the technology policy of the Ministry of 
Information and Communications. He is an Associate Professor at the Department of Industry Business at Korea University of Technology and Education. His research is focused on IT policy, R\&D evaluation, innovation management and technology planning.

Minseok CHOI received his PhD in Management Technology from the Korea Advanced Institute of Science and Technology (KAIST) in 2012. He has served as a Senior Researcher at ETRI (Electronics and Telecommunications Research Institute) for over 10 years. His research is focused on network theory and management information system.

Chanhoo SONG earned his PhD in Management from University of Nebraska, Chanhoo Song, taught at Fairleigh Dickinson University and Information and Communications University. His research interests include within and between group cooperative/competitive behaviours, turnover behaviours, competency modelling and evaluation, human resources management in R\&D organizations, and IT policy.

Kwanyoung KIM majored in IT Management in Korea Advanced Institute of Science and Technology (KAIST). He taught at Ulaanbaatar University, Mongolia and researched Policy and Strategy Development of IT R\&D and HRD. He is interested in joint research with developing countries in the field of ICT in education. He is especially focusing on International Development Cooperation (IDC) project in terms of technology transfer and education quality. 